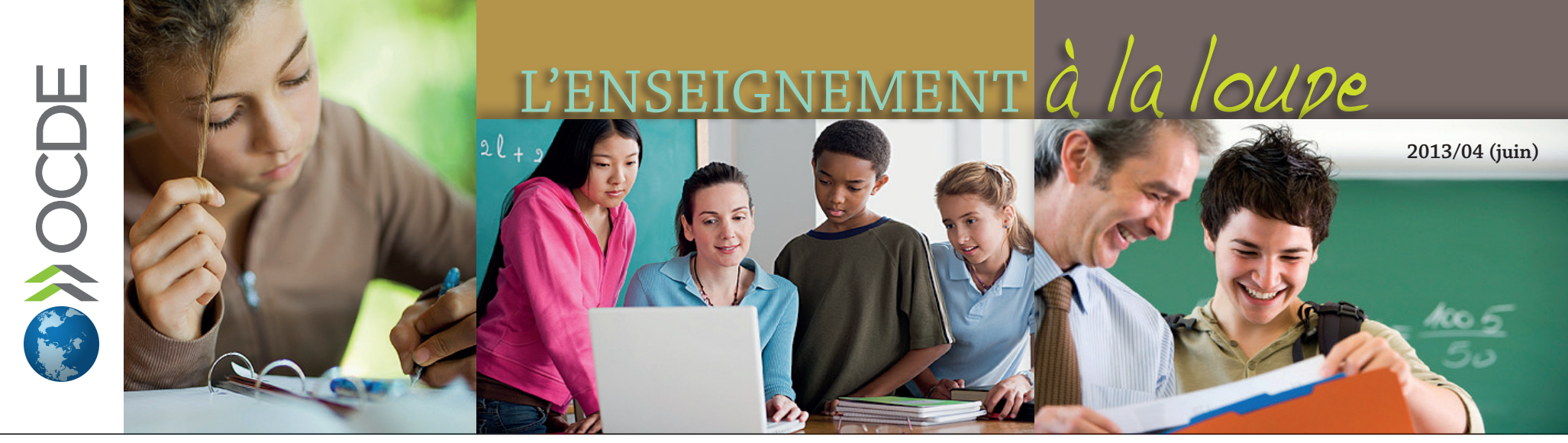

\title{
Encourager la création de communautés d'apprentissage parmi les enseignants
}

- D'après l'Enquête internationale sur l'enseignement et l'apprentissage (TALIS), les enseignants de tous les pays font massivement part de leur souhait d'avoir accès à davantage de formation continue.

- Dans tous les pays participant à l'enquête TALIS, les taux de formation continue de type coopératif et de pratiques pédagogiques collaboratives sont faibles.

- Les pays pourraient mettre la formation continue au service de la création efficace de communautés d'apprentissage professionnel au sein des établissements, et de leur renforcement.

TALIS, gu'est-ce gue c'est?

TALIS est la première enquête internationale s'intéressant aux environnements d'enseignement et d'apprentissage dans les établissements d'enseignement. L'enquête interroge des enseignants et des chefs d'établissement sur leur travail, leur établissement et leur(s) classe(s). Grâce à son analyse comparative internationale, elle aide les pays à identifier d'autres pays confrontés à des enjeux similaires, leur permettant ainsi de s'inspirer de leurs politiques respectives.

L'édition 2008 de l'enquête TALIS portait sur les enseignants du premier cycle du secondaire et leurs chefs d'établissement. L'enquête a été menée dans 200 établissements de 24 pays, au sein desquels 20 enseignants et le chef d'établissement ont été interrogés. L'enquête TALIS 2013 est actuellement en cours d'administration dans 33 pays.

Les données de cette note de synthèse proviennent principalement d'un rapport publié en 2012 intitulé Teaching Practices and Pedagogical Innovation: Evidence from TALIS. Pour tout complément d'information, consulter www.oecd.org/talis.

\section{Expérience des enseignants en matière de formation continue}

L'enquête TALIS interroge les enseignants sur leur accès à la formation continue, leur expérience en la matière, et leur perception de son incidence sur leur évolution en tant qu'enseignants. Parmi les pays à l'étude, le taux moyen de participation des enseignants à la formation continue varie. Dans l'ensemble, les enseignants affichent des taux plus élevés de participation à des activités de formation continue de type ateliers ou séminaires, par comparaison avec des activités de formation continue de type plus coopératif (voir le graphique 1). La formation continue de type coopératif est plus interactive et personnalisée que les ateliers ou les séminaires, dans la mesure où elle implique des activités comme l'observation ou la visite d'autres établissements, la participation à des réseaux d'enseignants avec un objectif commun, le tutorat, et/ou l'observation collégiale et le coaching.

En moyenne, 65 \% des enseignants ayant pris part à l'enquête TALIS indiquent participer à des activités de formation continue de type ateliers ou séminaires, contre $34 \%$ seulement pour les activités de formation continue de type coopératif ${ }^{1}$. Les différences de taux de participation à ce second type de formation continue sont toutefois marquées entre les pays : dans sept pays (le Portugal, l'Espagne, la Belgique [Fl.], l'Italie, l'Autriche, Malte et le Danemark), les enseignants font part de taux de participation inférieurs à $25 \%$, tandis que seuls deux pays (la Corée et l'Islande) enregistrent des taux de participation supérieurs à $50 \%$.

1. Pour calculer les échelles, on a fait la somme moyenne des items individuels associés à ces échelles. 


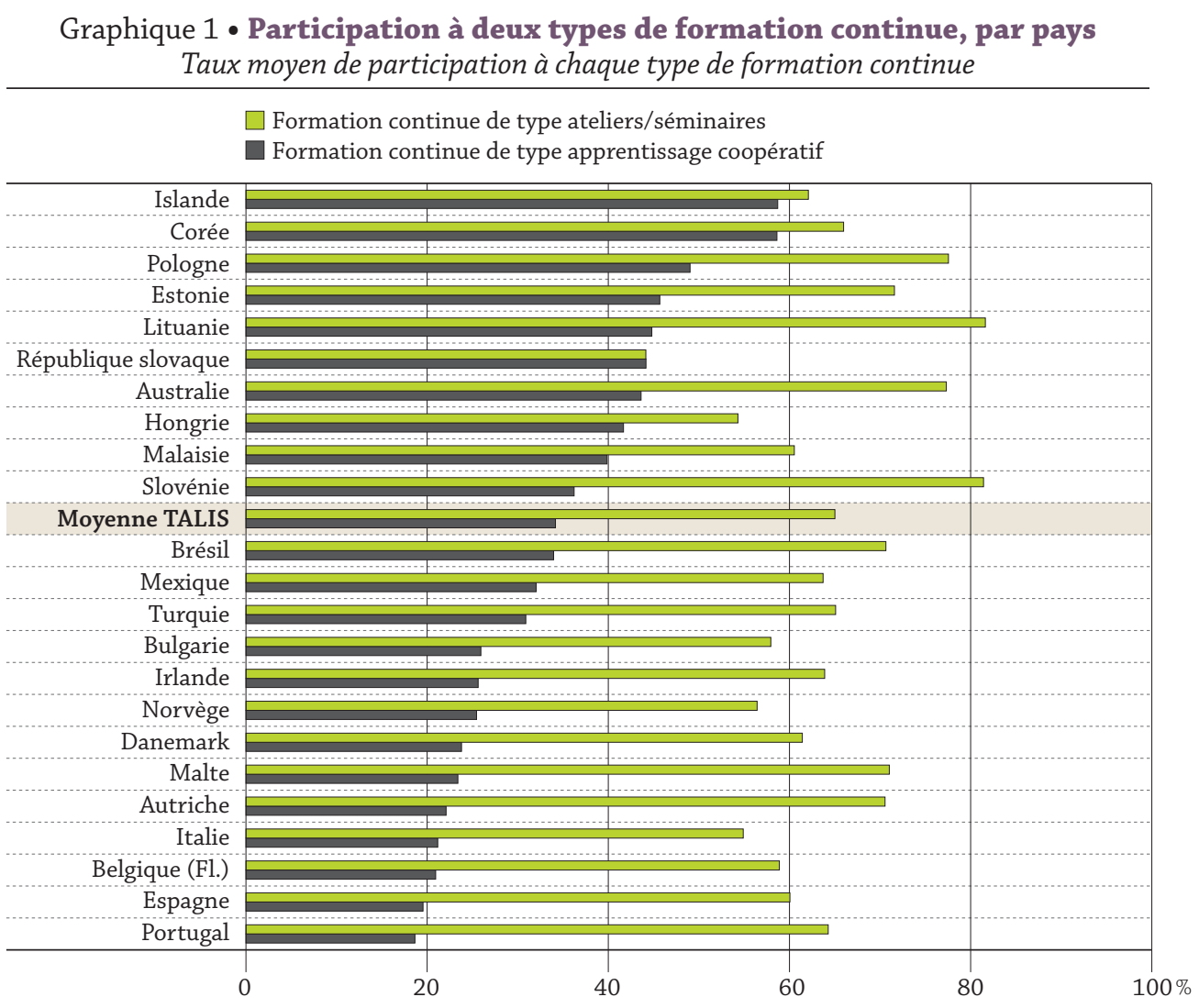

Source : OCDE, Base de données TALIS. Enquête internationale 2008 sur l'enseignement et l'apprentissage.

\section{Demande de davantage de formation continue}

Dans l'ensemble, les enseignants indiquent avoir besoin de davantage de formation continue. D'après les résultats de l'enquête TALIS', 55 \% des enseignants des pays à l'étude déclarent vouloir participer à davantage d'activités de formation continue qu'ils n'ont eu l'occasion de le faire au cours des 18 mois précédant l'enquête. Dans certains pays, ce taux est même plus élevé, plus de trois quarts des enseignants se disant ainsi dans ce cas au Mexique (85 \%), au Brésil (84 \%), en Malaisie (83 \%) et au Portugal (76 \%). La communauté flamande de Belgique est le seul participant où moins d'un tiers des enseignants disent vouloir participer à davantage d'activités de formation continue que celles auxquelles ils ont déjà accès ${ }^{3}$.

L'enquête TALIS interroge également les enseignants sur les types d'obstacles les empêchant de prendre part à davantage d'activités de formation continue. Dans les pays participants, les obstacles les plus fréquemment cités sont une incompatibilité avec l'emploi du temps professionnel de l'enseignant (47\%) et l'absence, selon les enseignants, d'une offre adéquate d'activités de formation continue (42\%).

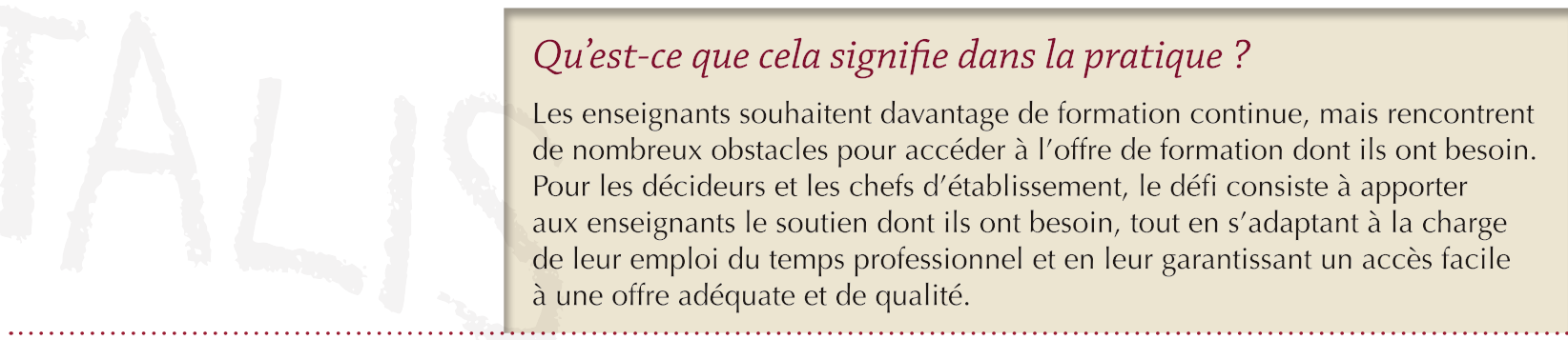

2. Rapport TALIS 2008 : Creating Effective Teaching and Learning Environments: First Results from TALIS.

3. TALIS 2008, tableau 3.3. 


\section{Émergence de communautés d'apprentissage professionnel}

Selon la définition retenue par Hord $(1997)^{4}$, les communautés d'apprentissage professionnel peuvent se définir comme des communautés formées à l’échelle des établissements et « visant l'amélioration continue des pratiques pédagogiques par l'implication du personnel dans des activités approfondies, systématiques et collaboratives de formation continue au niveau de l'établissement ». Les communautés d'apprentissage professionnel instaurent une culture de l'établissement promouvant l'apprentissage des élèves grâce à l'établissement de valeurs, de normes et d'attentes communes parmi les enseignants ${ }^{5}$.

L'enquête TALIS pose aux enseignants six questions reflétant les caractéristiques inhérentes aux communautés d'apprentissage professionnel. Les items retenus pour définir ces caractéristiques correspondent à la mesure dans laquelle il existe, dans l'établissement de l'enseignant, une vision commune, un attachement à l'apprentissage, une démarche de réflexion, une désindividualisation des pratiques, et des activités de collaboration. En théorie, lorsqu'un enseignant indique mettre en œuvre les cinq dimensions listées dans le tableau 1 ci-après, on peut alors considérer qu'il participe à une communauté d'apprentissage professionnel.

\section{Tableau 1. Libellé des items de l'échelle des communautés d'apprentissage professionnel}

\begin{tabular}{l|l} 
Dimension & $\begin{array}{l}\text { Question posée aux enseignants : à quelle fréquence mettez-vous en ouvre } \\
\text { les dimensions suivantes dans cet établissement ? }\end{array}$ \\
\hline \multirow{2}{*}{ Vision commune } & $\begin{array}{l}\text { Assister à des réunions du personnel afin de discuter de la vision et de la mission } \\
\text { de l'établissement. }\end{array}$ \\
\hline Attachement à l'apprentissage & Garantir l'application de normes communes pour l'évaluation des progrès des élèves. \\
\hline Réflexion & Participer à des activités d'apprentissage professionnel (par ex., encadrement d'équipe). \\
\hline Désindividualisation des pratiques & Observer les classes d'autres enseignants et leur faire part de commentaires. \\
\hline \multirow{2}{*}{ Activités de collaboration } & Échanger du matériel pédagogique avec des collègues. \\
\cline { 2 - 2 } & Pratiquer le co-enseignement au sein d'une même classe. \\
\hline
\end{tabular}

Source : OCDE (2012), Teaching Practices and Pedagogical Innovation: Evidence from TALIS, Éditions OCDE, tableau 3.2, p. 55.

D’après les données de l'enquête TALIS, dans de nombreux pays, les activités de collaboration sont courantes au sein du personnel, contrairement à la participation à une démarche de réflexion et à la désindividualisation des pratiques, bien moins répandues. La recherche se fait également l'écho de cette tendance ; les communautés d'apprentissage professionnel se sont répandues dans de nombreux pays, mais la création de ce type de communautés s'opère trop souvent dans le cadre d'une approche conventionnelle et descendante, et non suivant un processus plus ascendant et adapté au contexte ${ }^{6}$.

\section{Mettre la formation continue au service de la création de communautés d'apprentissage professionnel}

Il existe des liens étroits entre la formation continue et les communautés d'apprentissage professionnel dans tous les pays participant à l'enquête TALIS. En premier lieu, la formation continue, notamment celle de type coopératif, est particulièrement propice à l'instauration, parmi les enseignants, des valeurs, normes et attentes communes qui caractérisent les communautés d'apprentissage professionnel. L'analyse des données de l'enquête TALIS (voir le graphique 2) révèle que les enseignants participant à des activités de formation continue de type apprentissage coopératif présentent également un taux plus élevé de participation aux communautés d'apprentissage professionnel au sein de leur établissement, par comparaison avec les enseignants participant à des activités de formation continue de type ateliers ou séminaires. (Seule la Norvège échappe à ce constat.) Dans 19 des pays à l'étude, l'association entre communautés d'apprentissage professionnel et formation continue de type coopératif est même souvent plus marquée qu'avec le sentiment d'efficacité personnelle, pourtant également connu pour son lien étroit avec ce type de communautés.

4. OCDE (2012), Teaching Practices and Pedagogical Innovation: Evidence from TALIS, Éditions OCDE, p. 34. Voir Hord, S.M. (1997), Professional Learning Communities: Communities of Continuous Inquiry and Improvement, Southwest Educational Laboratory, Austin, Texas.

5. Bryk, A.S. et M.E. Driscoll (1988), The High School as Community: Contextual Infuences and Consequences for Students and Teachers, National Center on Effective Schools, University of Wisconsin - Madison, Madison, Wisconsin.

6. OCDE (2012), Teaching Practices and Pedagogical Innovation: Evidence from TALIS, Éditions OCDE, p. 33. 


\section{Graphique 2 • Association entre d'une part, deux types de formation continue et le sentiment d'efficacité personnelle des enseignants, et d'autre part, les communautés d'apprentissage professionnel, par pays}

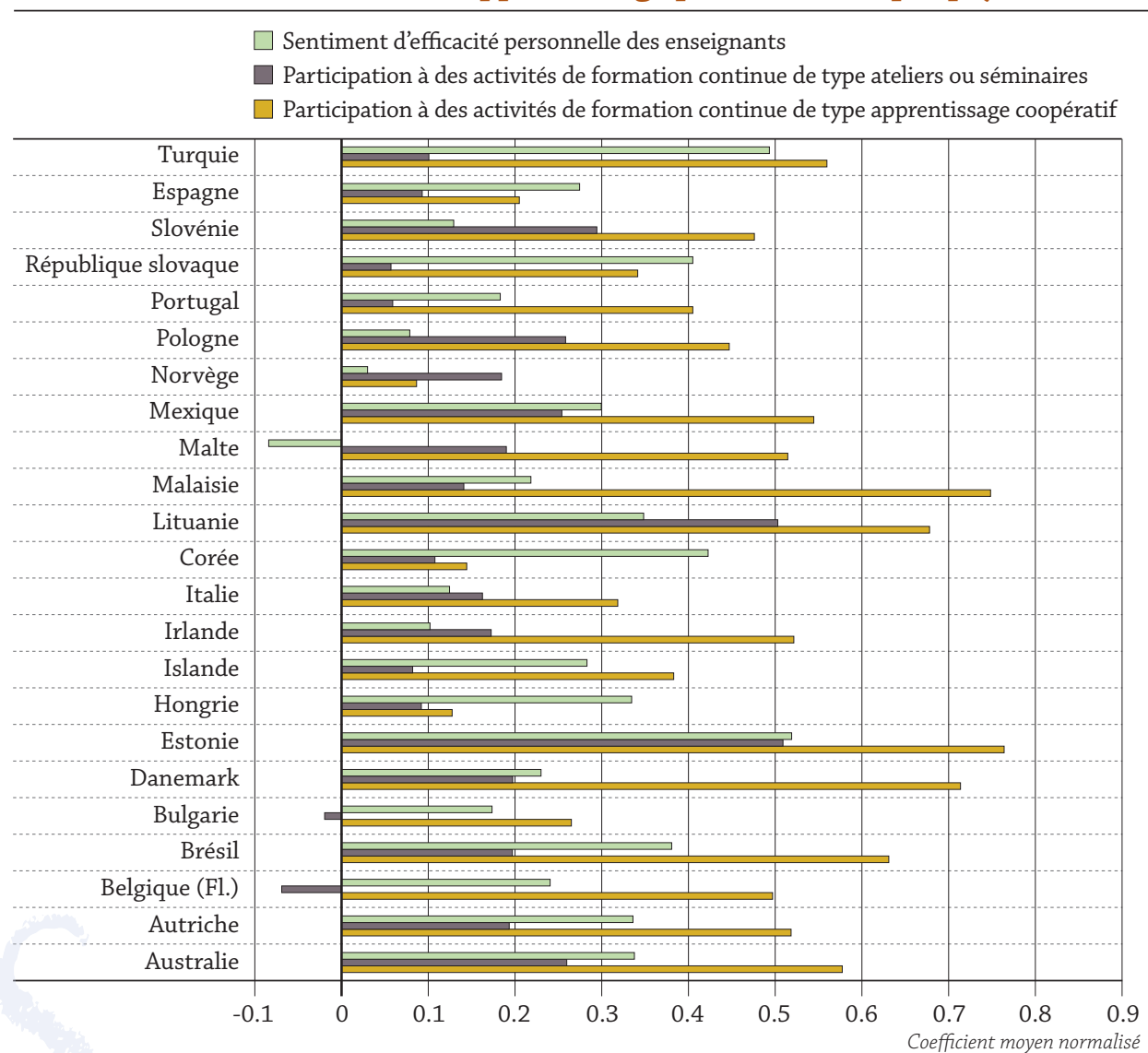

Source : OCDE, Base de données TALIS. OCDE (2012), Teaching Practices and Pedagogical Innovation: Evidence from TALIS, Éditions OCDE, annexe B, pp. 131-143.

Pour conclure Les communautés d'apprentissage professionnel formées au sein des établissements peuvent apporter, à l'échelle de tout l'établissement, un soutien continu et adapté au contexte de chaque enseignant. Les enseignants souhaitant davantage de formation continue, mais rencontrant souvent des difficultés pour trouver des offres compatibles avec leur emploi du temps et leur apportant le soutien adéquat au moment opportun, le développement d'activités de formation continue de type coopératif recevrait un accueil favorable dans le corps enseignant et pourrait, dans le même temps, favoriser la création de communautés d'apprentissage professionnel à l'échelle des établissements.

\section{Voir}

www.oecd.org/talis
Contacter

Kristen Weatherby (Kristen.Weatherby@oecd.org)
Pour en savoir plus

OCDE (2012), Teaching Practices and Pedagogical Innovation: Evidence from TALIS, Éditions OCDE, Paris.

Ce document est publié sous la responsabilité du Secrétaire général de l'OCDE. Les opinions qui y sont exprimées et les arguments qui y sont employés ne reflètent pas nécessairement les vues officielles des pays membres de l'OCDE.

Ce document et toute carte qu'il peut comprendre sont sans préjudice du statut de tout territoire, de la souveraineté s'exerçant sur ce dernier, du tracé des frontières et limites internationales, et du nom de tout territoire, ville ou région.

Les données statistiques concernant Israël sont fournies par et sous la responsabilité des autorités israéliennes compétentes. L'utilisation de ces données par l'OCDE est sans préjudice du statut des hauteurs du Golan, de Jérusalem-Est et des colonies de peuplement israéliennes en Cisjordanie aux termes du droit international. 